\title{
Food Quality Monitoring Based on Hydrolysis-Induced Au-Catalyzed Heck Cross-Coupling by Ag Metallization
}

\author{
Sanaz Gholampour, ${ }^{1}$ Hossein Jalali $\left(\mathbb{D},{ }^{1}\right.$ Rahele Zhiani $\mathbb{D}^{2},{ }^{2}$ Hassan Rashidi $\left(\mathbb{D},{ }^{3}\right.$ \\ and Alireza Motavalizadehkakhky $\mathbb{D}^{2}$ \\ ${ }^{1}$ Department of Food Science and Technology, Damghan Branch, Islamic Azad University, Damghan, Iran \\ ${ }^{2}$ New Materials Technology and Processing Research Center, Department of Chemistry, Neyshabur Branch, Islamic Azad University, \\ Neyshabur, Iran \\ ${ }^{3}$ Food Industries Department, Khorasan Razavi agricultural and Natural Resources Research and Education Center, AREEO, \\ Mashad, Iran
}

Correspondence should be addressed to Hossein Jalali; drmagjalali@yahoo.com and Rahele Zhiani; r_zhiani2006@yahoo.com

Received 11 October 2020; Revised 2 April 2021; Accepted 7 May 2021; Published 26 May 2021

Academic Editor: Eduard Llobet

Copyright (c) 2021 Sanaz Gholampour et al. This is an open access article distributed under the Creative Commons Attribution License, which permits unrestricted use, distribution, and reproduction in any medium, provided the original work is properly cited.

\begin{abstract}
Visual detection of meat spoilage was performed based on hydrolysis-induced silver metallization on gold nanoparticles (Au NPs). The hydrolysis of 4-I-benzene-bounded Wang resin was induced by the release of a biogenic amine followed by Au-catalyzed Heck cross-coupling reaction that made silver-coated gold core-shell NPs (Au@Ag) in the presence of Ag ions (Ag metallization). A portable sensory cap was designed by this hypothesis and the successful results were obtained for histamine, trimethylamine, and a spoilage sheep meat. With this protocol, the localized surface plasmon resonance (LSPR) is tuned for absorption of Au NPs and leads to LSPR peak blue shift of gold nanoparticles due to the Ag metallization and the preparation of Au@Ag coreshell NPs. Au NPs and the resulting Au@Ag NPs were characterized by transmission electron microscopy (TEM), BET, ultraviolet-visible (UV-Vis), X-ray diffraction (XRD), energy dispersive X-ray (EDX), and dynamic light scattering (DLS) analyses. Also, various control experiments were set up to credit the portable sensory tube.
\end{abstract}

\section{Introduction}

The foodborne illness caused by pathogens, toxins, and other contaminants is a serious risk to human health. Conventional methods for detecting pathogenic bacteria and toxins are time consuming and relatively difficult, requiring special laboratory equipment and experienced operator [1-3]. Nanomaterials, including metal oxides as well as metal nanoparticles, carbon nanotubes, and quantum dots, play an important role in the design of sensors and biosensors for food analysis [4]. Metallic nanoparticles made of gold or silver have many visual and electronic properties depend on their size and composition. Because these materials have a high affinity for bonding with biomolecules, they can be used to create chemical sensors [5-9]. Electronic properties (absorption, emission), light scattering, and conductivity of nanomaterials can be changed or modified. In recent years, much research has been done on the design of nanoscale materials to identify pathogenic bacteria $[2,4]$. Gold nanoparticles have been widely used for various sensors due to their unique properties $[1,10]$. Chemicals on the nanoparticle surface can be controlled by organic bond-forming agents such as thiol molecules or thiol-containing polymers, resulting in a high particle affinity for bonding with sulfhydryl groups (which form relatively strong covalent bonds) [1, 11]. Further modification of the surface of gold nanoparticles can be performed by molecules with thiolated functional groups, binding to various probes including antibodies and nucleic acids [4]. The proper formation of gold nanoparticles is associated with the presence of a surface plasmon group with a visible/ultraviolet absorption profile [12]. The surface plasmon group results from the accumulation of electrons 
attached to the conductive group due to the small particle size. The change in the surface plasmon group depends on the particle size, the surrounding chemicals, the particle surface adsorption capacity, and their dielectric constant $[1,10]$. Local changes in the dielectric current of nanoparticles, by adsorption of biomolecules or induction of biomarkers of nanoparticles, cause changes in localized surface plasmon resonance (LSPR) [13]. These unique properties of gold as well as silver allow nanoparticles to be used for several different types of detection processes using changes in the surface plasmon resonance. In this way, any change causes a change in the absorption of the spectrum caused by visible/ultraviolet rays $[12,14]$. In addition to absorption, gold nanoparticles are used as an indicator to detect and determine multiple targets using fluorescence, Raman scattering, electrical conductivity, atomic force, and magnetic force techniques $[15,16]$.

Biogenic amines are low molecular weight organic compounds that are often produced by the decarboxylation of free amino acids [17]. This decarboxylation removes the $\alpha$ carboxyl group from the amino acid and produces biogenic amines such as histamine from histidine, tyramine from tyrosine, and tryptophan from tryptophan [16, 18]. Availability of free amino acids, high processing temperatures or the presence of decarboxylase-producing microorganisms and favorable conditions for microbial growth and decarboxylase activity, storage temperature, ripening time, and packaging are among the prerequisites for the biogenic amine formation in food $[19,20]$. Free amino acids are present in foods that are released as a result of proteolytic activity or thermal degradation of proteins [21]. The chemical structure of these amines is aliphatic putrescine, aromatic tyramine, and heterocyclic histamine. Consumption of excessive amounts of spoiled meat (containing biogenic amines) will have toxic effects such as headaches, diarrhea, respiratory problems, and heart palpitations [16, 22]. Due to their high importance on the health of living organisms, as well as the high cost of devices and the cost of analysis, various sensors have been developed to detect biogenic amines, which can be referred to PVA electrospun nanofibers containing Ag NPS [13], porous PVA and GO [15], Zn(II) complex-based optical sensor [16], Genipin [18], polydiacetylene nanofibers [23], functional aromatic polyamides with sensory pendant groups toward amines [18], and naphthyl end-capped terthiophene [22]. Recently, Lapenna et al. exploited naked $\mathrm{Au}$ NPs to colorimetric detection of histamine as a biogenic amine [1]. In this way, colorimetric detection of biogenic amines is a safe promising way to visual monitoring of food spoilage [24]. This strategy provides a versatile, nondestructive, free instrument, and cheap approach for the efficient detection of food quality. Colorimetric sensors are simple and easy work-up and can be directly read out by bare eyes $[25,26]$.

Previously, Lin et al. developed a colorimetric detection of biogenic amines, based on hydrolysis-induced Ag metallization of Au nanorods (NRs) to tune the PLSR (localized surface plasmon resonance) absorption of Au NRs [10]. In this work, Au-catalyzed hydrolysis-induced Heck coupling Ag metallization of Au NPs was served for the detection of biogenic amines released from spoilage sheep meat. Moreover, the protocol was simulated by histamine as a biogenic amine and trimethylamine to mimic a real volatile biogenic amine for the validation of the protocol. The main principle of this protocol is based on the integration of hydrolysis-induced silver metallization by the as-generated biogenic amines released from a spoilage meat. This biogenic amine induced a Heck cross-coupling reaction followed by formation of $\mathrm{Au} @ \mathrm{Ag}$ core-shell NPs in the presence of Ag ions. Based on the intrinsic LSPR property of Au NPs, that is, depends on their size, and composition, and shape, formation of Au@Ag NPs provides a colorimetric detection of food spoilage.

\section{Materials and Methods}

2.1. Instrumentation. Electronic spectra (UV-Vis analyses) were performed on a UV Spectrolab BEL photonics. GCMS analyses were conducted on an Agilent gas chromatography/mass spectrometer (GC/MS) system supplied with the detectors of Agilent technologies 6890 N Network GC system and Agilent Technologies 5973 mass-selective. The size distribution of the Au and Au@Ag nanoparticles was measured by dynamic light scattering (DLS) method using a HORIBALB550 instrument. The X-ray diffraction (XRD) analyses of the nanoparticles were recorded on a Bruker AXS D8advance $\mathrm{X}$-ray diffractometer using $\mathrm{Cu}-\mathrm{K} \alpha$ radiation. Transmission electron microscopy (TEM) images were performed using a Philips EM208 microscope operated at $100 \mathrm{kV}$. The surface area of the obtained Au and Au@Ag NPs was determined by $\mathrm{N}_{2}$ physisorption at $-196^{\circ} \mathrm{C}$ on a Micromeritics ASAP 2000 instrument using the BET method. Energydispersive X-ray (EDX) spectroscopy was done on a field emission scanning electron microscope (FE-SEM), JEOL $7600 \mathrm{~F}$, equipped with a spectrometer for energy dispersion of X-rays from Oxford instruments.

2.2. Materials and Methods. All materials were supplied from Sigma and Fluka suppliers and used as received without any further purification. Ultrapure solvents with analytical grade were used for all experiments. Wang resin (polymerbounded 4-iodobenzene) was purchased from Novabiochem containing 0.64-1.1 mmol loading, 100-300 mesh, and crosslinked with $1 \%$ divinylbenzene.

2.3. Preparation of $A u$ NPs. Gold nanoparticles were prepared according to a previously reported protocol [5]. Briefly, in first, $125 \mathrm{~mL}$ of deionized water was boiled in a $250 \mathrm{~mL}$ round bottom flask. Then, a $1 \%$ solution of $\mathrm{HAuCl}_{4}$ $(2.0 \mathrm{~mL})$ was added to the mixture and the resulting solution was stirred for $2.0 \mathrm{~min}$. Sodium citrate $(10.0 \mathrm{~mL}, 0.05 \mathrm{M})$ as a reducing agent was added dropwise (during $10.0 \mathrm{~min}$ ) with continuous stirring and under reflux conditions. The color of the solution was changed from pale yellow to deep red at around $10 \mathrm{~min}$ indicating the successful preparation of $\mathrm{Au}$ $\mathrm{NPs}$, where $\mathrm{Au}$ (III) ions is reduced to metallic $\mathrm{Au}(0)$ with the red color appearance [6]. The as-prepared Au NPs were stored as a solution at $4^{\circ} \mathrm{C}$ after cooling the reaction mixture.

The stability of the Au NPs was monitored every day (up to 10 days) using UV-Vis techniques based on the characteristic peak $\lambda_{\max }$ of the Au NPs. 


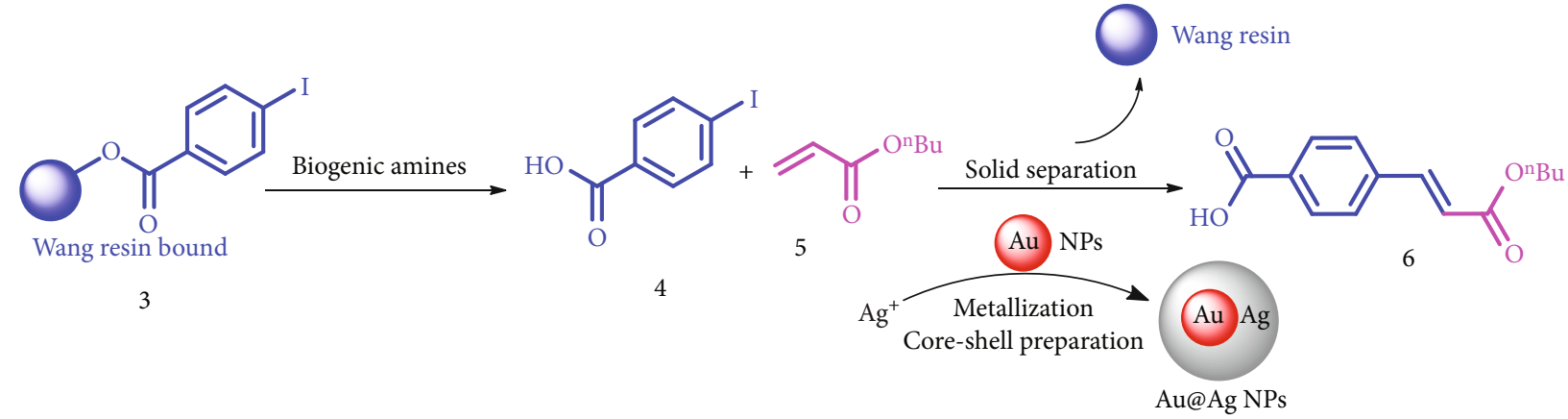

Scheme 1: The mechanism of detection of food spoilage based on induced hydrolysis due to release of biogenic amines and subsequent coupling reaction with gold nanoparticles and silver metallization.

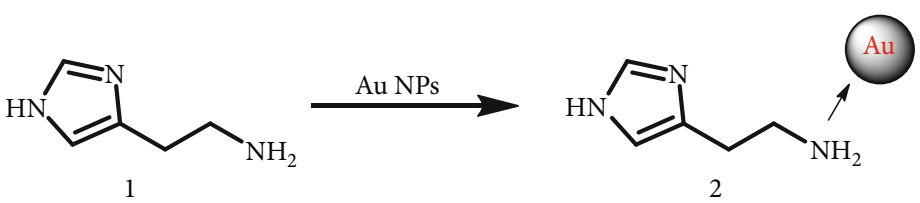

Scheme 2: Efficient coordination of Au NPs with histamine [4].

2.4. Colorimetric Detection Simulation of Gold Nanoparticles Using Histamine as a Control Test. To mimic the hydrolysis of the reaction of 4-iodobenzoic acid from a Wang resin in the presence of a released biogenic amine from a spoilage food, histamine, as a biogenic amine, was used. The Suzuki coupling reaction is catalyzed by Au NPs in the presence of $n$-butyl acrylate, and then, silver metallization causes the preparation of Au@Ag NPs. For this goal, Au NP solution $(25 \mu \mathrm{L}), \mathrm{AgNO}_{3}$ solution $(3 \mu \mathrm{L}, 50 \mathrm{mM}), 4$-iodobenzenebounded Wang resin, and histamine solution $(50 \mu \mathrm{L}, 1 \mathrm{M})$ were mixed and homogenized. The resulting mixture was incubated for 0.5 hour at $30^{\circ} \mathrm{C}$. The absorption spectrum of the solution was recorded by a UV-Vis instrument over $400-850 \mathrm{~nm}$. The resulting Suzuki coupling product was detected and identified over a GC-MS instrument.

\subsection{Visual Sensing of Released Biogenic Amines by Portable} Sensory Hydrogels. Visual detection of biogenic amines was performed according to the protocol reported by Lin et al. [10]. The snap cap of the $1.5 \mathrm{~mL}$ polypropylene microentrifuge tube was charged with the following mixture: In first, $20 \mathrm{mg}$ of agarose was dissolved in $2.0 \mathrm{~mL}$ of boiling water. The solution was allowed to cool to $40^{\circ} \mathrm{C}$; then, 4 iodobenzene-bounded Wang resin, $200 \mu \mathrm{L}$ of solution of $\mathrm{Au}$ NPs, and $\mathrm{AgNO}_{3}(20 \mu \mathrm{L}, 05 \mathrm{mM})$ were added to the agarose solution and homogenized. The snap cap of the microcentrifuge tube was charged by $100 \mu \mathrm{L}$ of the mixture solution, and the resulting sensory hydrogel was solidified after $5 \mathrm{~min}$ at room temperature. The sensory was used to the detection of a solution of trimethylamine (TMA, a high volatile compound with a low B.P.). As shown in Scheme $1,0.5 \mathrm{~mL}$ ethanolic solution of trimethylamine was added to the bottom of microcentrifuge tube that its snap cap was previously loaded with the sensory hydrogel [10]. The snap cap was closed, and the tube was kept in upright position at room temperature. The color change of the snap cap sensory hydrogel results of the vapour of TMA was recorded as pictures taken by a smartphone camera.

Finally, for the monitoring of food spoilage, $5.0 \mathrm{~g}$ of a fresh sheep meat, as a real sample, was added to the microcentrifuge tube with a sensory snap cap under identical conditions described for TMA analysis. A bare microcentrifuge with a sensory snap cap was used as a reference. The color change of the sensory snap cap was recorded as pictures taken by a smartphone camera.

\section{Results and Discussion}

As a hypothesis presented by Lin et al. [10], histamine, as a biogenic amine, not only increases the $\mathrm{pH}$ of the medium and hydrolyzes 4-iodobenzoic acid from the Wang resin but also catalyzes the subsequent Suzuki coupling reaction by coordinating gold nanoparticles (Scheme 2). Histamine also plays a base role in the coupling reaction. The coupling reaction catalyzed by gold-histamine nanoparticles, due to the oxidative-addition, reductive-elimination steps of the coupling reaction, causes the reduction of silver ions in the medium (metallization) and the subsequent formation of Au@Ag core-shell nanoparticles. Coating of gold nanoparticles with silver metallization increases the surface-to-volume ratio of gold nanoparticles causing a blue shift in the LSPR longitudinal peak, which results in a distinct color change from pink to brick red. The rate of color change and blue shift of the longitudinal LSPR peak is directly related to the concentration of biogenic amines and the degree of spoilage of the meat.

Scheme 1 shows the mechanism of detection of food spoilage based on induced hydrolysis due to the release of biogenic amines and the subsequent reaction of catalyzed coupling with gold nanoparticles and silver metallization.

To investigate the validity of the hypothesis and the effect of biogenic amines on the designed model, first, the 


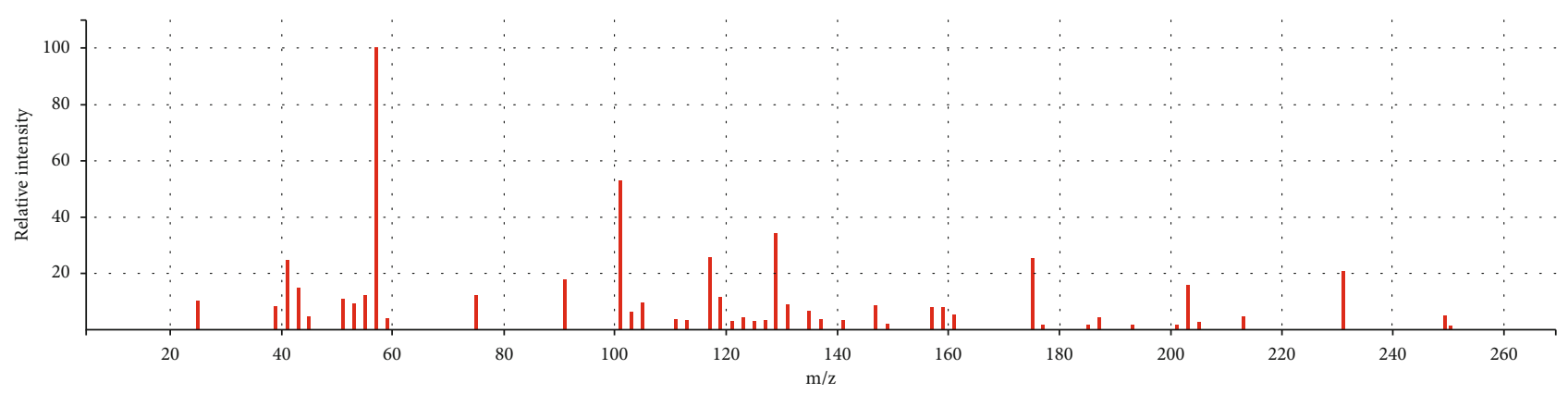

FiguRE 1: MS spectrum (in situ from solution) of 4-(3-butoxy-3-oxoprop-1-en-1-yl) benzoic acid (6).

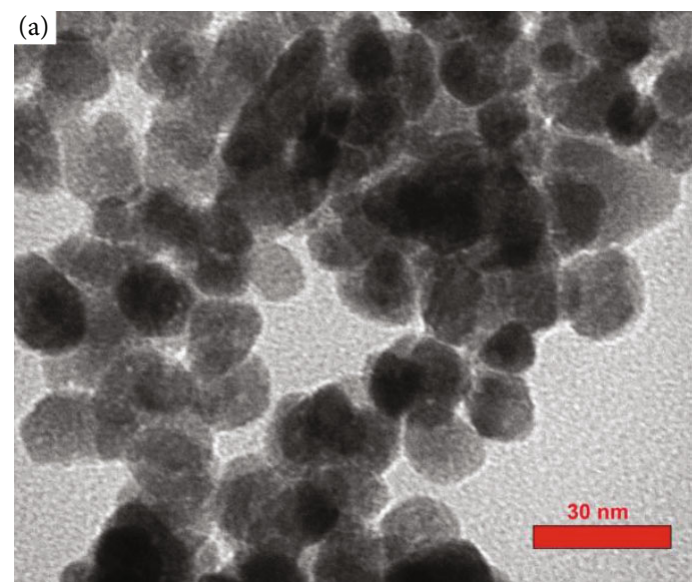

(a)

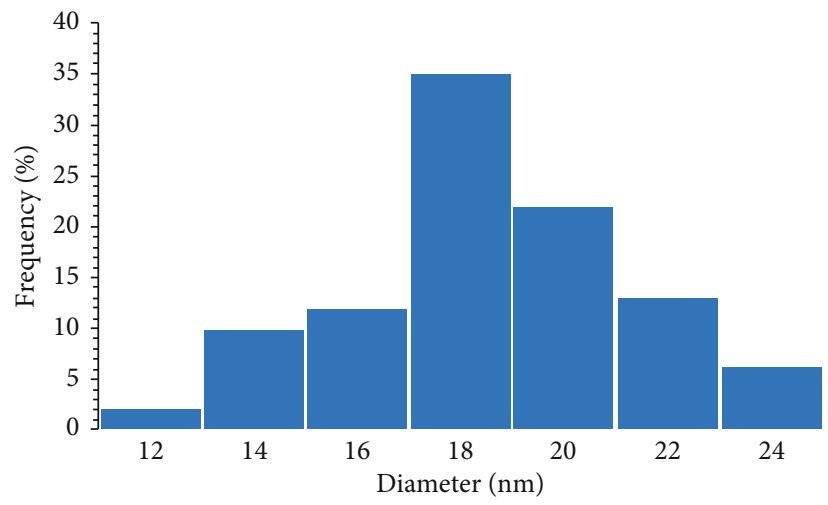

(c)

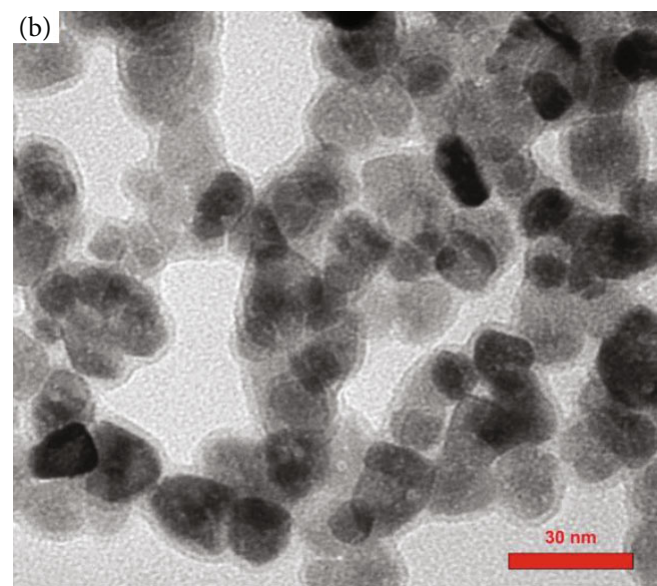

(b)

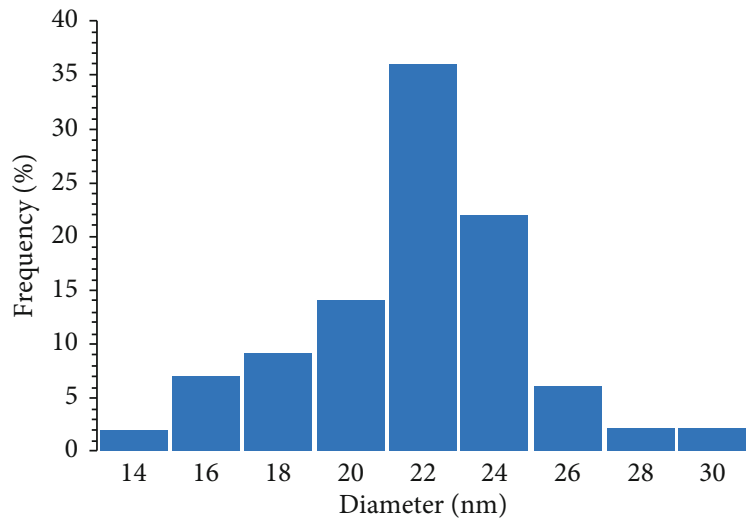

(d)

Figure 2: TEM images and the corresponding DLS analyses of $(a, c)$ the freshly prepared Au NPs and (b, d), after the exposure of TMA vapour(Au@Ag NPs).

hydrolysis-induced Au-catalyzed Suzuki reaction of 4iodobenzene-bounded Wang resin with $n$-butyl acrylate was performed in the presence of $\mathrm{Ag}^{+}$ions and histamine as a biogenic amine. The progress of the reaction was monitored by GC-MS as well as UV-Vis techniques.

GC-MS analysis showed the formation of the coupling product 4-(3-butoxy-3-oxoprop-1-en-1-yl) benzoic acid 6, with a molecular mass of $249 \mathrm{~g} \cdot \mathrm{mol}^{-1}$ from the hydrolyzed 4-iodobenzoic acid and $n$-butyl acrylate (Figure 1).

In addition, characterization of the nanoparticle, before and after the reaction, also shows a good Au@Ag core-shell preparation in the reaction, which is a strong proof of the hydrolysis of 4-iodobenzoic acid from Wang resin and the subsequent coupling reaction by gold nanoparticles and silver metallization, and finally, the preparation is $\mathrm{Au} @ \mathrm{Ag}$ core-shell NPs.

3.1. Characterization of Au NPs: Before and after Food Spoilage Monitoring Experiment. The as-prepared Au NPs were characterized by EDX, TEM, XRD, BET, and DLS analyses. Also, the resulting NPs over the reaction of hydrolysisinduced Au-catalyzed Heck cross-coupling by $\mathrm{Ag}$ 


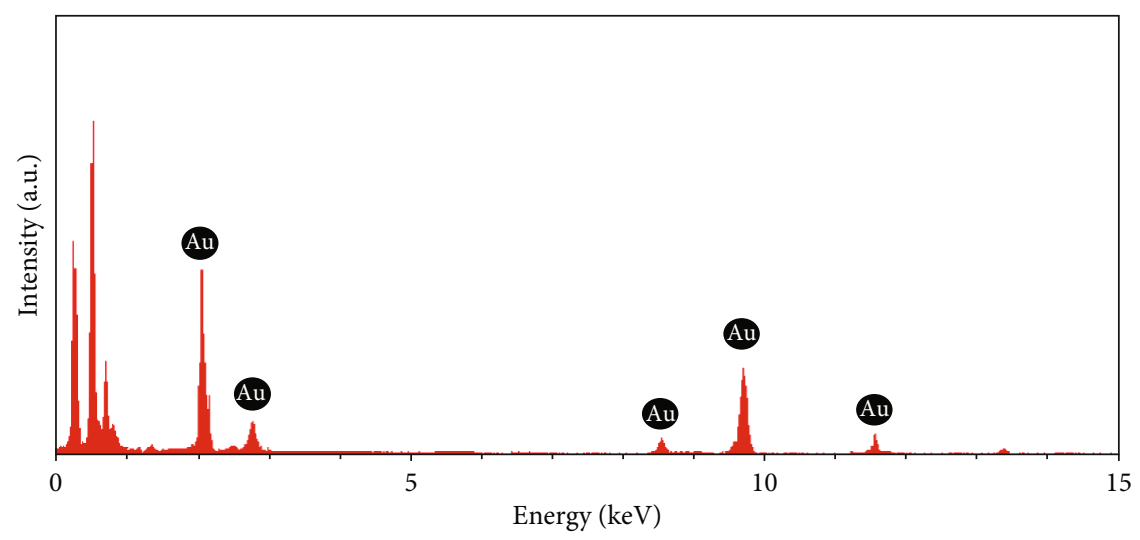

(a)

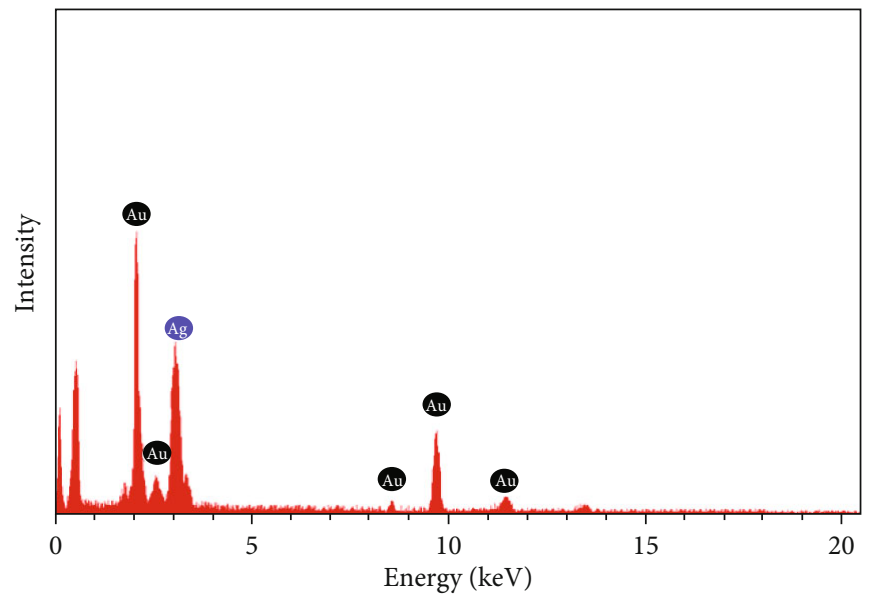

(b)

Figure 3: EDX analyses of (a) the freshly prepared Au NPs and (b) after the exposure of TMA vapour (Au@Ag NPs).

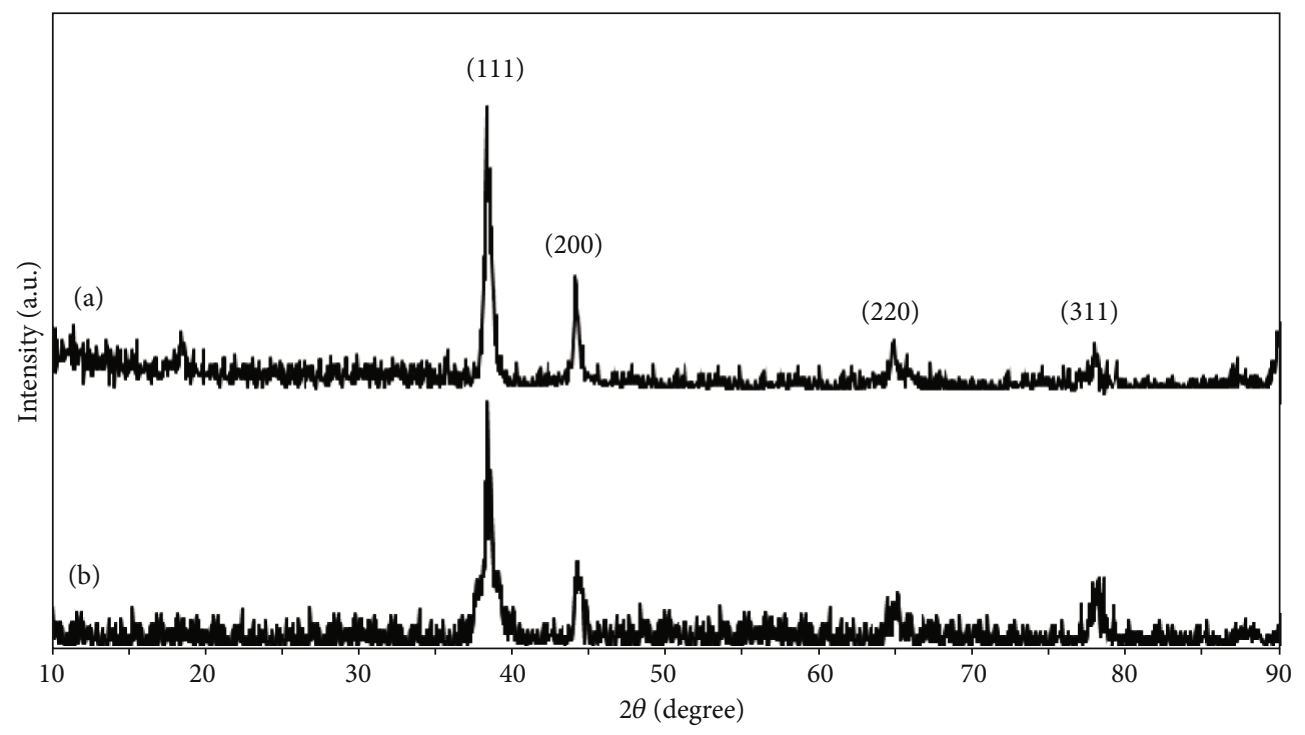

FIGURE 4: XRD patterns of (a) freshly prepared Au NPs and (b) after the exposure of TMA vapour (Au@Ag NPs).

metallization in the presence of histamine were characterized (Figures 2-4).

As shown in Figure 2(a), gold nanoparticles have a spherical shape with a diameter of about 14 nanometers. In addi- tion, nanoparticles have a homogeneous and uniform morphology. An increase in size of about $2 \mathrm{~nm}$ in the TEM image of Au@Ag nanoparticles also well indicates the formation of a silver shell on the gold nanoparticles (Figure 2(b)) 


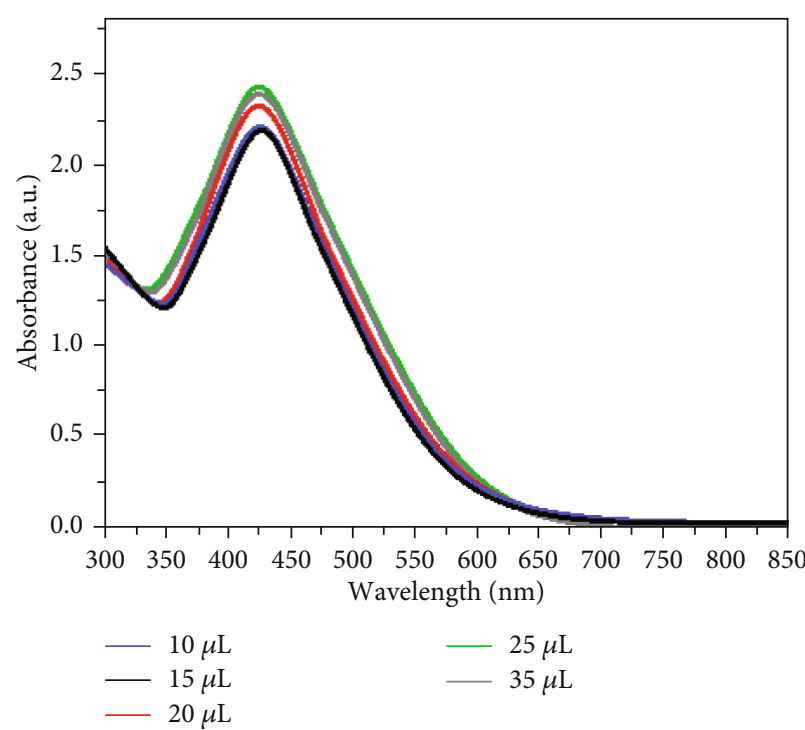

(a)

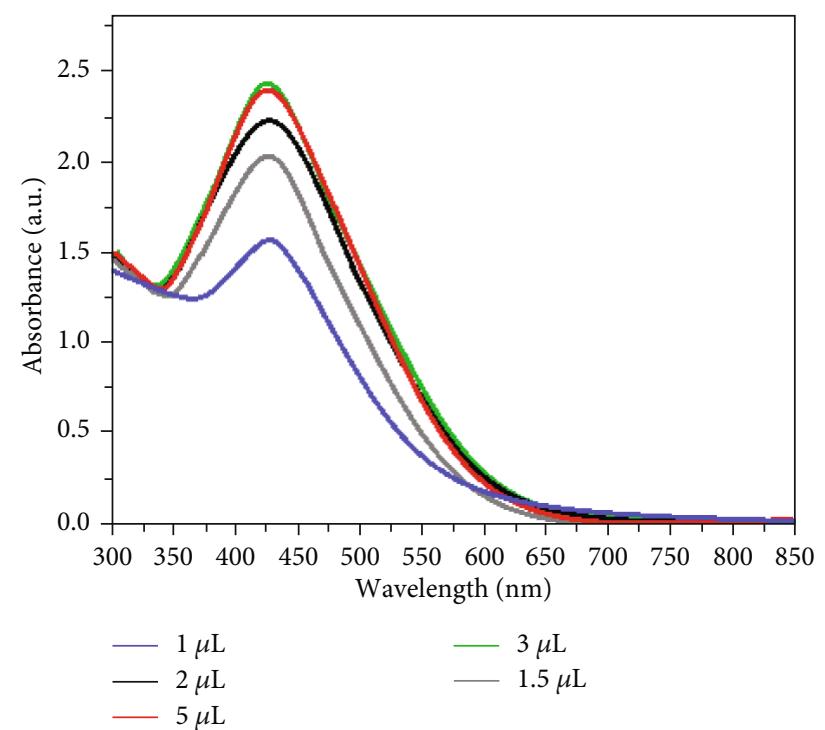

(b)

FIgURE 5: Effect of (a) Au NP concentration in $3 \mu \mathrm{L} \mathrm{AgNO}_{3}$ solution and (b) Ag ion concentration in $25 \mu \mathrm{L}$ aqueous solution of Au NPs for the hydrolysis-induced Au-catalyzed Heck cross-coupling by Ag metallization in the presence of histamine. For each study, all other conditions were kept constant.

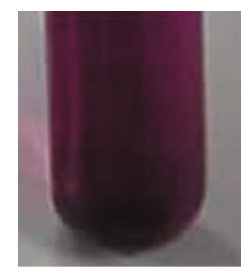

10

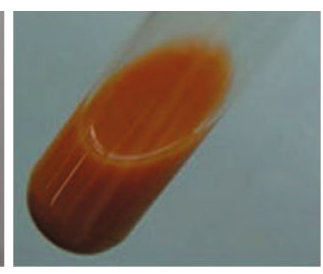

20

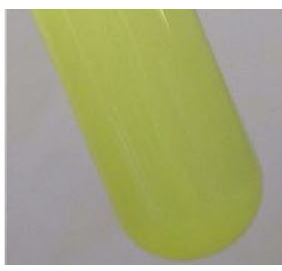

30

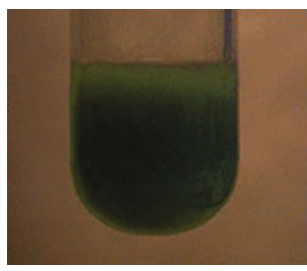

40

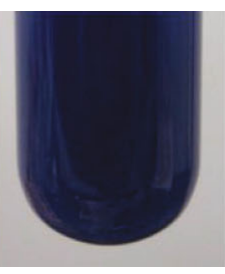

45

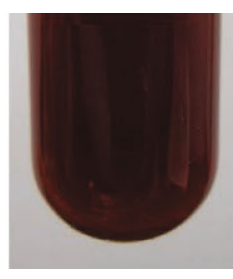

50

Figure 6: Bare eye detectable color changes of an aqueous mixture of $\mathrm{Au} \mathrm{NPs/AgNO} /$ histamine/4-I-benzene-bounded Wang resin as a function of time.

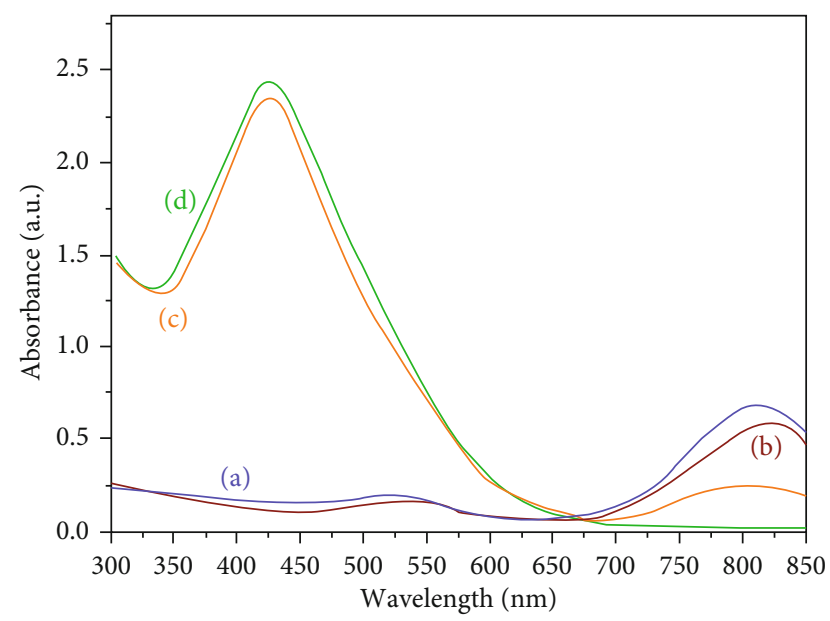

Figure 7: UV-Vis spectra of (a) Au NPs, (b) aqueous mixture of Au NPs-histamine, (c) aqueous mixture of $\mathrm{Au}$ $\mathrm{NPs} / \mathrm{AgNO}_{3} /$ histamine/4-I-benzene-bounded Wang resin, and (d) isolated Au@Ag NPs.
$[14,27]$. The silver shell is also more pronounced with a lighter background in Au@Ag particles in the corresponding TEM image. The particle size distribution, in full agreement with TEM analysis, shows the average particle sizes of 18 and $22 \mathrm{~nm}$ for gold and Au@Ag nanoparticles, respectively (Figures 2(c) and 2(d)). According to BET analysis, gold and Au@Ag nanoparticles have a specific surface area of 21.2 and 18.6 square meters per gram, respectively. The decrease in the specific surface area of gold nanoparticles was also quite consistent with the increase in nanoparticle diameter (TEM image and DLS analyses), indicating the metallization of silver and the formation of $\mathrm{Au} @ \mathrm{Ag}$ nanoparticles.

EDX analysis also shows the formation of gold and Au@Ag nanoparticles with the corresponding binding energies (Figure 3(a)). The peaks appearing at 2.1, 2.7, 8.5, 9.7, 11.5 , and $13.3 \mathrm{eV}$ correspond to the binding energies of the electrons at different levels of $\mathrm{Au}$ (in both spectra), and the peaks appearing at $3.0 \mathrm{eV}$ in the EDX spectrum of $\mathrm{Au} @ \mathrm{Ag}$ nanoparticles also belonging to the Ag particles are coated in Au nanoparticles (Figure 3(b)). 


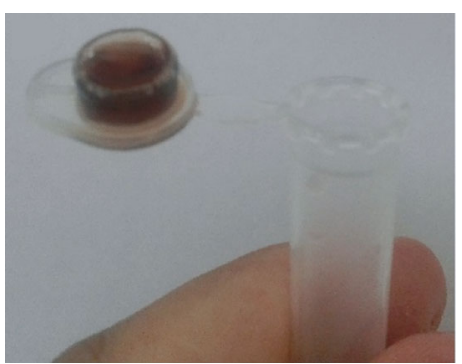

(a)

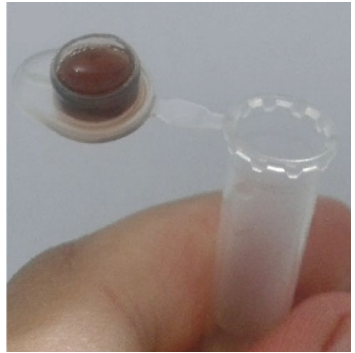

(b)

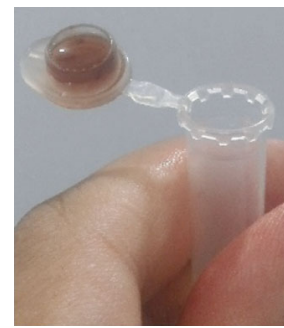

(c)

Figure 8: Images of (a) a snap cap hydrogel-loaded microcentrifuge tube after exposure of TMA solution, (b) after exposure of a fresh sheep meat sample, and (c) after exposure of a spoilage sheep meat sample.

Figure 4 shows the X-ray diffraction pattern of $\mathrm{Au}$ spherical nanoparticles and Au@Ag core-shell nanoparticles. Gold nanoparticles have 4 characteristic peaks at $2 \theta$ $=38.2^{\circ}, 44.4^{\circ}, 64.8^{\circ}$, and $77.7^{\circ}$ which belong to the planes (111), (200), (220), and (311), respectively. The X-ray diffraction pattern of the nanoparticles is fully consistent with the lattice structure of the face-centered cubic (FCC) (Figure 4(a)) $[27,28]$. The X-ray diffraction pattern of Au@Ag nanoparticles also has characteristic peaks at 2 $\theta=38.2^{\circ}, 44.4^{\circ}, 64.8^{\circ}$, and $77.7^{\circ}$, which indicate that the crystal structure of gold nanoparticles does not change during the silver metallization and the formation of Au@Ag (Figure 4(b)). Only a significant amount of crystalline peaks has been reduced, which in itself confirms the formation of Au@Ag core-shell NPs (Figure 4(b)).

3.2. Optimization of Au NPs and Ag Ion Amount for the Hydrolysis-Induced Au-Catalyzed Heck Cross-Coupling by Ag Metallization. In order to achieve the highest absorption in the reaction of the hydrolysis-induced Au-catalyzed Heck cross-coupling by Ag metallization in the presence of histamine and subsequently observe the visual detection (color change) of the solution (or in the prepared sensor), the amount of gold nanoparticles and silver ion concentration was optimized. The absorption intensity was measured at different concentrations of gold nanoparticles and reaches the highest absorption intensity at $25 \mu \mathrm{L}$ (Figure 5(a)). As shown in Figure 5(b), the LSPR peak decreases at concentrations higher and lower than $25 \mu \mathrm{L}$. The concentration of silver ions was much more noticeable than the concentration of gold nanoparticles (compare spectra (a) with (b)), so that a decrease in its concentration reduces the peak intensity of LSPR, and at $3 \mu \mathrm{L}$ of silver ions, maximum absorption occurs. The results will show the silver metallization on gold nanoparticles.

Figure 6 shows the color change of the aqueous mixture of $\mathrm{Au} \mathrm{NPs} / \mathrm{AgNO}_{3} /$ histamine/4-I-benzene-bounded Wang resin over time. As shown in the figure, it shows 6 distinct colors recognizable with naked eyes including purple, orange, yellow, green, blue, and brick red at 50-minute intervals. As mentioned earlier, the amount of color change depends on the concentration of amines released in the solution (or the amount of amines released by spoilage meat).

Figures 7(a)-7(d) show UV-Vis analysis of silver and $\mathrm{Au}$ @Ag nanoparticles in different solutions. Gold nanoparticles have poor absorption at $535 \mathrm{~nm}$ and are red in color
(Figure 7(a)). This electron transfer, in the presence of the biogenic amine histamine, undergoes a red shift that indicates the coordination of nanoparticles to histamine, as shown in Scheme 2 (Figure 7(b)). Figure 7(c) shows the in situ UV-Vis spectrum from the aqueous mixture of $\mathrm{Au}$ $\mathrm{NPs} / \mathrm{AgNO}_{3} /$ histamine/4-I-benzene-bounded Wang resin. Strong absorption at $426 \mathrm{~nm}$ is a characteristic of Au@Ag nanoparticles and shows that silver metallization is well performed on gold nanoparticles and leads to LSPR peak blue shift of gold nanoparticles $[2,5,6,27]$. In addition, the presence of a peak in the visible region of $803 \mathrm{~nm}$ belonging to the gold nanoparticles indicates that the gold nanoparticles are still present in the mixture and are not completely coated by the silver particles. Figure 7(d) also shows the electronic absorption of Au@Ag nanoparticles isolated from the reaction mixture in a pure form.

Then, a sensor prepared with a snap cap loaded microcentrifuge tube was used to observe the visual detection of the hydrogel placed in the snap cap, which was exposed to the atmosphere of TMA vapour, spoilage meat, and histamine. Figure 8 shows the results after 50 minutes of exposure to each of them. Visible color change clearly shows the effect of biogenic amines and consequently the quality of the meat.

3.3. Control Experiments. In order to investigate the catalytic effect of gold nanoparticles, the coupling reaction of $n$-butyl acrylate with 4-iodobenzene-bounded Wang resin in the presence of silver ions and histamine and in the absence of Au nanoparticles was analyzed. The results of GC-MS analysis showed no efficiency for the coupling product 6 . The catalytic activity of gold nanoparticles also confirms the hypothesis based on the metallization of silver ions and the preparation of Au@Ag core-shell NPs. In another experiment, Au@Ag core-shell nanoparticles were prepared by chemical method according to the procedure presented by Samal et al. [29]. Then, the above reaction was performed in the presence of $\mathrm{Au} @ \mathrm{Ag}$ nanoparticles as a catalyst (in the absence of gold nanoparticles and silver ions). The results were completely similar to the previous analysis, and no efficiency was observed for the mating product.

\section{Conclusion}

A new versatile, nondestructive, free instrument, and cheap approach has been developed for the efficient detection of 
food quality by bare eyes through the solidification of a smart sensor on a microcentrifuge snap cap. The system is based on the hydrolysis-induced Au-catalyzed Heck cross-coupling by Ag metallization results of exposure of a vapour of biogenic amines released from a spoilage meat. Three following successful tests validated the protocol: (1) a simulated control in the presence of histamine to mimic act of a biogenic amine, (2) exposure of the hydrogel sensor loaded in a snap cap of a micro-centrifuge tube to TMA solution, and (3) examination of the detection tube with a real spoilage sheep meat. All tests revealed the successful visual detection of released biogenic amines with a blue shift of longitudinal LSPR related to $\mathrm{Au}$ NPs in the result of $\mathrm{Ag}$ metallization followed by Au@Ag core-shell preparation. This change could be visual to bare eyes, which makes the method for the promising fast detection of meat quality. Moreover, characterization of Au NPs and the resulting Au@Ag NPs confirmed their structure and subsequently the mechanism proposed for the detection of biogenic amines. The method demonstrated several advantages including sensitive, instrument-free, and high-resolution colorimetric detection for the end-users due to the distinctive multiple color readout, while most of the previous methods suffer from the problems of being expensive, destructive, nonreproducibility, and tedious work-up. The proposed method is superior to many previously reported methods in terms of simplicity, ease of use, repeatability, and high stability in order to evaluate the quality of meat, so that it becomes a reliable alternative to previously reported methods, slowly.

\section{Data Availability}

The data that support the findings of this study are available in the supplementary material of this article.

\section{Conflicts of Interest}

The authors declare that they have no conflict of interest.

\section{Acknowledgments}

This work was supported by the research council of Islamic Azad University, Damghan branch.

\section{References}

[1] A. Lapenna, M. Dell'Aglio, G. Palazzo, and A. Mallardi, ""Naked" gold nanoparticles as colorimetric reporters for biogenic amine detection," Colloids and Surfaces A: Physicochemical and Engineering Aspects, vol. 600, article 124903, 2020.

[2] S. Y. Tseng, S. Y. Li, S. Y. Yi, A. Y. Sun, D. Y. Gao, and D. Wan, "Food quality monitor: paper-based plasmonic sensors prepared through reversal nanoimprinting for rapid detection of biogenic amine odorants," ACS Applied Materials \& Interfaces, vol. 9, no. 20, pp. 17306-17316, 2017.

[3] K. Sowoidnich, H. Schmidt, H. D. Kronfeldt, and F. Schwägele, "A portable $671 \mathrm{~nm}$ Raman sensor system for rapid meat spoilage identification," Vibrational Spectroscopy, vol. 62, pp. 70-76, 2012.
[4] F. Mustafa and S. Andreescu, "Nanotechnology-based approaches for food sensing and packaging applications," RSC Advances, vol. 10, no. 33, pp. 19309-19336, 2020.

[5] K. M. A. El-Nour, E. T. A. Salam, H. M. Soliman, and A. S. Orabi, "Gold nanoparticles as a direct and rapid sensor for sensitive analytical detection of biogenic amines," Nanoscale Research Letters, vol. 12, no. 1, article 2014, pp. 231-311, 2017.

[6] T. Amir, A. Fatma, and A. Hakan Hacettepe, "Synthesis and characterization of gold nanoparticles as a sensing tool for the lateral flow immunoassay development," Hacettepe Journal of Biology and Chemistry, vol. 1, pp. 100051-100226, 2020.

[7] S. Lv, K. Zhang, Q. Zhou, and D. Tang, "Plasmonic enhanced photoelectrochemical aptasensor with D-A F8BT/g- $\mathrm{C}_{3} \mathrm{~N}_{4}$ heterojunction and AuNPs on a 3D-printed device," Sensors and Actuators B: Chemical, vol. 310, article 127874, 2020.

[8] G. Cai, Z. Yu, R. Ren, and D. Tang, "Exciton-plasmon interaction between AuNPs/graphene nanohybrids and CdS quantum dots/TiO2for photoelectrochemical aptasensing of prostatespecific antigen," ACS Sensors, vol. 3, no. 3, pp. 632-639, 2018.

[9] J. Zhuang, W. Lai, M. Xu, Q. Zhou, and D. Tang, "Plasmonic AuNP/g-C3N4 nanohybrid-based photoelectrochemical sensing platform for ultrasensitive monitoring of polynucleotide kinase activity accompanying DNAzyme-catalyzed precipitation amplification," ACS Applied Materials \& Interfaces, vol. 7, no. 15, pp. 8330-8338, 2015.

[10] T. Lin, Y. Wu, Z. Li, Z. Song, L. Guo, and F. Fu, "Visual monitoring of food spoilage based on hydrolysis-induced silver metallization of au nanorods," Analytical Chemistry, vol. 88, no. 22, pp. 11022-11027, 2016.

[11] R. Abargues, P. J. Rodriguez-Canto, S. Albert, I. Suarez, and J. P. Martínez-Pastor, "Plasmonic optical sensors printed from Ag-PVA nanoinks," Journal of Materials Chemistry C, vol. 2, no. 5, pp. 908-915, 2013.

[12] C. F. Chow, "Biogenic amines- and sulfides-responsive gold nanoparticles for real-time visual detection of raw meat, fish, crustaceans, and preserved meat," Food Chemistry, vol. 311, article 125908, 2020.

[13] C. Marega, J. Maculan, G. Andrea Rizzi et al., "Polyvinyl alcohol electrospun nanofibers containing Ag nanoparticles used as sensors for the detection of biogenic amines," Nanotechnology, vol. 26, no. 7, article 075501, 2015.

[14] T. Yaseen, H. Pu, and D. W. Sun, "Rapid detection of multiple organophosphorus pesticides (triazophos and parathionmethyl) residues in peach by SERS based on core-shell bimetallic Au@Ag NPs," Food Additives \& Contaminants: Part A, vol. 36, no. 5, pp. 762-778, 2019.

[15] T. Siripongpreda, K. Siralertmukul, and N. Rodthongkum, "Colorimetric sensor and LDI-MS detection of biogenic amines in food spoilage based on porous PLA and graphene oxide," Food Chemistry, vol. 329, article 127165, 2020.

[16] M. A. Sahudin, M. S. Su'ait, L. L. Tan, Y. H. Lee, and N. H. Abd Karim, "Zinc (II) salphen complex-based fluorescence optical sensor for biogenic amine detection," Analytical and Bioanalytical Chemistry, vol. 411, no. 24, article 2025, pp. 6449-6461, 2019.

[17] R. Torre, E. A. Costa-Rama, H. Nouws, and C. Delerue-Matos, "Screen-printed electrode-based sensors for food spoilage control: bacteria and biogenic amines detection," Biosensors, vol. 10, no. 10, p. 139, 2020.

[18] I. Mallov, F. Jeeva, and C. B. Caputo, "An edible genipin based sensor for biogenic amine detection," Journal of Chemical Technology \& Biotechnology, 2020. 
[19] X. Zhai, Z. Li, J. Shi et al., "A colorimetric hydrogen sulfide sensor based on gellan gum-silver nanoparticles bionanocomposite for monitoring of meat spoilage in intelligent packaging," Food Chemistry, vol. 290, pp. 135-143, 2019.

[20] N. Kaur, S. Chopra, G. Singh et al., "Chemosensors for biogenic amines and biothiols," Journal of Materials Chemistry $B$, vol. 6, no. 30, pp. 4872-4902, 2018.

[21] L. González-Ceballos, B. Melero, M. Trigo-López et al., "Functional aromatic polyamides for the preparation of coated fibres as smart labels for the visual detection of biogenic amine vapours and fish spoilage," Sensors and Actuators B: Chemical, vol. 304, article 127249, 2020.

[22] Q. Liu, S. Mukherjee, R. Huang et al., "Naphthyl end-capped terthiophene-based chemiresistive sensors for biogenic amine detection and meat spoilage monitoring," Chemistry-An Asian Journal, vol. 14, no. 15, pp. 2751-2758, 2019.

[23] M. Valdez, S. K. Gupta, K. Lozano, and Y. Mao, "ForceSpun polydiacetylene nanofibers as colorimetric sensor for food spoilage detection," Sensors and Actuators B: Chemical, vol. 297, article 126734, 2019.

[24] R. Zeng, J. Wang, Q. Wang, D. Tang, and Y. Lin, "Horseradish peroxidase-encapsulated DNA nanoflowers: an innovative signal- generation tag for colorimetric biosensor," Talanta, vol. 221, article 121600, 2021.

[25] R. Ren, G. Cai, Z. Yu, Y. Zeng, and D. Tang, "Metal-polydopamine framework: an innovative signal-generation tag for colorimetric immunoassay," Analytical Chemistry, vol. 90, no. 18, pp. 11099-11105, 2018.

[26] R. Ren, G. Cai, Z. Yu, and D. Tang, "Glucose-loaded liposomes for amplified colorimetric immunoassay of streptomycin based on enzyme-induced iron(II) chelation reaction with phenanthroline," Sensors and Actuators B: Chemical, vol. 265, pp. 174-181, 2018.

[27] Z. Xu, Y. Hou, and S. Sun, "Magnetic core/shell $\mathrm{Fe}_{3} \mathrm{O}_{4} / \mathrm{Au}$ and $\mathrm{Fe}_{3} \mathrm{O}_{4} / \mathrm{Au} / \mathrm{Ag}$ nanoparticles with tunable plasmonic properties," Journal of the American Chemical Society, vol. 129, no. 28, pp. 8698-8699, 2007.

[28] D. Philip, "Biosynthesis of Au, Ag and Au-Ag nanoparticles using edible mushroom extract," Spectrochimica Acta Part A: Molecular and Biomolecular Spectroscopy, vol. 73, no. 2, pp. 374-381, 2009.

[29] A. K. Samal, L. Polavarapu, S. Rodal-Cedeira, L. M. Liz-Marzán, J. Pérez-Juste, and I. Pastoriza-Santos, "Size tunable $\mathrm{Au@Ag} \mathrm{core-shell} \mathrm{nanoparticles:} \mathrm{synthesis} \mathrm{and} \mathrm{surface-}$ enhanced Raman scattering properties," Langmuir, vol. 29, no. 48, pp. 15076-15082, 2013. 